\title{
Pathological dislocation of the hip due to coxotuberculosis in children: a 29-case report
}

\author{
Xin Jiang, Yuan Li', Lijun Liu, MingXin Peng, XueYang Tang, DaoXi Wang and XiaoDong Yang
}

\begin{abstract}
Background: The aim of this study is to evaluate the clinical outcome of various surgery methods in children suffering pathological dislocation of the hip joint due to coxotuberculosis.

Patients and methods: From January 2006 to January 2011, 29 children with coxotuberculosis hip dislocation were treated with open reduction (4 children) and acetabular reconstruction (25 children). According to degree of dislocation and age, acetabular reconstructions included Salter osteotomy (nine children), Pemberton operation (six children), Dega operation (seven children), the hip shelf arthroplasty (two children), and Chiari operation (one child). During acetabular reconstruction, 16 children underwent upper femoral rotational shortening osteotomies simultaneously, 6 children underwent the femoral head and neck reconstruction simultaneously, and 3 children were underwent corrective osteotomy. After operation, children were immobilized with plaster external fixation for 1-3 months and treated with routine anti-infective therapy for 3 days. During follow-up study (12-18 months), the hip stability was examined via X-ray. The recovery was evaluated via acetabular index (Al) and Harris hip score.
\end{abstract}

Results: Postoperative X-ray films of 29 children showed concentric reduction of all hips. Total 25 children healed I incision surgery, while 4 children with skin antrum of incision were further treated leading to 1-3 months delay of healing. Total 24 children were followed up for $2-5$ years. X-ray examination revealed no redislocation after 2 years postoperative. The postoperative Al was normal $\left(15^{\circ}-20^{\circ}\right)$ in 22 children and increased to $25^{\circ}-30^{\circ}$ in 2 children. After 2 years postoperative, 8 children had normal function of hip joint, 13 children had mild limitation of flexion and rotation, and 3 children had fibrous ankylosis. The average of Harris hip score was 83 (ranged, 62-90).

Conclusion: In our study, pathological hip dislocations of children attributed to coxotuberculosis were treated via open reduction and acetabular reconstruction and the outcomes were both excellent. Individual characteristic should be taken into consideration during treatment, and proper surgery approach should be adopted according to pathological changes of the hip.

Keywords: Pathological hip dislocation, Coxotuberculosis, Open reduction, Acetabular reconstruction

\section{Introduction}

Pathological dislocation of the hip joint in children is relatively rare occurrence and may cause lifelong disability when missed or ill-treated. The hip is a deep-seated articulation, and its dislocation may either remain hidden for some time or go undiagnosed [1]. According to the development of hip dislocation, pathological dislocation of the hip is frequently associated with heavy bone destructive defect of acetabulum and femoral heads and necks as well as the residual anatomic deformity. Therefore, this

\footnotetext{
* Correspondence: yuanlilili@hotmail.com

Department of Pediatric Surgery, West China Hospital, Sichuan University, No. 37 Guoxue Road, Chengdu, Sichuan 610041, People's Republic of China
}

disease is difficult to treat, and several surgery operations have been explored and showed different outcomes.

Tuberculosis of the hip presents significant clinical problems, accounting for approximately $15 \%$ of osteoarticular tuberculosis [2]. The disease once occurred in the hip may lead to progressive destruction of the joint if untreated at an early stage, and many even proceed to pathological dislocation. Dislocated hips with infection are difficult to be managed to obtain a stable, mobile, congruous, and concentric joint. Diagnosis and effective treatment at an early stage are vital to treat the disease and save the joint [3]. The reports on the management of residual anatomic deformity of hip dislocation due to 
tuberculosis in children have rarely been documented. No comprehensive treatment protocol has been outlined.

From 2006 to 2011, we have performed surgery approaches in 29 children with pathological tubercular dislocation of the hip, accounting for $3.4 \%$ of hip dislocation in our hospital. The details of the patients' general data and clinical results are presented here.

\section{Patients and methods}

This study was approved by the Institutional Review Board of West China Hospital, Sichuan, China. Written informed consent was obtained from the patients for the publication of this report and any accompanying images. Based on the diagnosis card index at our department during the period from January 2006 to January 2011, we searched the files of patients with pathological hip dislocation caused by tuberculosis. Patients were included in the study if they met the following criteria: no previous treatment in other hospitals and radiographs available from the start of treatment to healing. Patients with slight subluxation or acetabular dysplasia were excluded.

Clinical manifestation of 29 patients with hip dislocation due to coxotuberculosis was shown in Table 1. Total 29 children (13 boys and 16 girls) with an average age of 4.3 years (ranged, 1-11 years) were included in the present study. There were 12 Han Chinese, 9 ethnic Tibetans, and 8 Yi people. Pathological tubercular hip dislocation is located in the right hip (17 children) and left hip (12 children). Four children showed subluxation of the hip joint, and 25 showed total dislocation of the hip. The mean duration of hip dislocation was 18 month (ranged, 10 days to 10 years). Besides the hip pain and lameness, other accompanied symptoms included significant local swelling in hip (13 children), fever $\left(38^{\circ} \mathrm{C}-39^{\circ} \mathrm{C}\right)$ ( 6 children), pulmonary tuberculosis ( 7 children), spinal tuberculosis ( 2 children), tibia tuberculosis ( 1 child), femoral tuberculosis ( 1 child), and iliac fossa abscess ( 1 child). The function status of injured hip joint was assessed using the Harris hip score $[4,5]$. The average of preoperative Harris hip score was 61 (ranged, 43-78). All children presented the increased erythrocyte sedimentation rate, and the PPD skin test was positive in 23 children. Preoperative $\mathrm{X}$-ray examination was performed for all children, and other examinations included computed tomography (CT) scan and three-dimensional reconstruction (23 children), color Doppler ultrasound (18 children), and magnetic resonance imaging (MRI) (12 children). The radiologic examination indicated the hip dislocation in all patients accompanied by avascular necrosis of femoral head ( 15 children), excalation of femoral head ( 6 children), total absence of femoral head ( 2 children), and absence of femoral head and neck ( 1 child). Additionally, eight children had increased anteversion angle $\left(40^{\circ}-60^{\circ}\right)$ and three had varus deformity. The acetabular index (AI) was measured
Table 1 Clinical manifestation of 29 patients with hip dislocation due to coxotuberculosis

\begin{tabular}{|c|c|}
\hline Clinical manifestation & Patients \\
\hline Patients (male/female) & $32(19 / 13)$ \\
\hline Age (years) & 4.3 (ranged, 1-11) \\
\hline Han/Tibetan/Yi nationality & $12 / 9 / 8$ \\
\hline Duration (months) & 18 (ranged, 10 days -10 years) \\
\hline \multicolumn{2}{|l|}{ Dislocation of hip joint } \\
\hline Subluxation & 4 \\
\hline Complete dislocation & 25 \\
\hline \multicolumn{2}{|l|}{ Concomitant symptom } \\
\hline Hip local swelling & 13 \\
\hline Fever $\left(38^{\circ} \mathrm{C}-39^{\circ} \mathrm{C}\right)$ & 6 \\
\hline Pulmonary tuberculosis & 7 \\
\hline Spondylocace & 2 \\
\hline Tibia tuberculosis & 1 \\
\hline Femoral tuberculosis & 1 \\
\hline Liac fossa abscess & 1 \\
\hline Harris score & $61(43-78)$ \\
\hline \multicolumn{2}{|l|}{ Acetabular index } \\
\hline $15-20^{\circ}$ (normal) & 11 \\
\hline $25-35^{\circ}$ (mild) & 12 \\
\hline $40-50^{\circ}$ (severe) & 6 \\
\hline \multicolumn{2}{|l|}{ Preoperative examination } \\
\hline Damage of acetabulum & 18 \\
\hline Ischemic necrosis of femoral head & 15 \\
\hline Excalation of femoral head & 6 \\
\hline Total absence of femoral head & 2 \\
\hline Absence of femoral head and neck & 1 \\
\hline
\end{tabular}

according to Kleinberg and Lieberman [6] on the primary radiographs. It was roughly normal in 11 patients with the values of $15^{\circ}-20^{\circ}$ and increased to $25^{\circ}-35^{\circ}$ in 12 patients and to $40^{\circ}-50^{\circ}$ in 6 patients.

\section{Surgical approaches}

For the management of active tuberculosis of the hip, all patients were given routine antituberculosis therapy for 2-4 weeks before surgery. After routine preoperative preparations, patients were placed in a supine position and the contralateral hip was raised. Then, a Salter anterior approach to the hip was performed after intubation and general anesthesia. Firstly, the hip lesion was removed thoroughly and histopathologically examined to make a definitive diagnosis.

Different operation methods were selected for 29 patients according to different pathological types. Four patients younger than age 2 were treated with open reduction and external fixation by plaster hip spica. Acetabular 
reconstruction was performed for 25 patients. Among them, nine patients aged less than 6 with the $\mathrm{AI}<40^{\circ}$ and femoral head congruent with the acetabula underwent Salter osteotomy. Six patients aged 3-6 with the AI $>40^{\circ}$, femoral head not congruent with the acetabula and unclosed Y-shaped acetabular cartilages were treated with Pemberton's operation. In addition, Dega operation was used for seven patients aged 5-14 years with total hip dislocation. The hip shelf arthroplasty was performed for two patients aged more than 14 years with hip subluxation. One patient aged more than 14 with complete hip dislocation was treated via Chiari operation.

During the acetabular reconstruction, 16 patients underwent upper femoral rotational shortening (short, 1-3 cm; mean, $2 \mathrm{~cm}$ ) osteotomies simultaneously. Six patients also underwent the femoral head and neck reconstruction, and their greater trochanter were moved down. Corrective osteotomy was performed in three patients to correct coax vara, and the neck-shaft angle was corrected to $25^{\circ}$ (ranged, $15^{\circ}-40^{\circ}$ ).

\section{Postoperative therapy}

All affected limbs underwent external fixation postoperatively by plaster cast for 1-3 months. To prevent additional infection, cephalosporins were given for 3 days after surgery, whilst all patients were given antituberculosis therapy for $12-18$ months. Postoperative X-ray photograph of each patient was taken. The exercise of patients was scheduled according to the stability of the hip joints and the healing of osteotomies.

\section{Results}

Pathological examination indicated that all 29 cases were hip dislocation due to coxotuberculosis. Postoperative X-ray films showed well hip reduction of all patients. Postoperatively, 25 patients healed I incision surgery, while 4 patients were accompanied by skin antrum, which were cut, and the healing was delayed for 1.5 months (ranged, 1-3 months).

A total of 24 patients were followed up for 3 years (ranged, 2-5 years) (Table 2). No complication was detected, such as hip redislocation, pathologic fracture and looseness of internal fixation during the follow-up period. X-ray examination revealed that no dislocation reoccurred at 2 years after operation. The postoperative AI was $15^{\circ}-20^{\circ}$ in 22 patients and increased mildly to $25^{\circ}-30^{\circ}$ in 2 patients. The anteversion angle was $20^{\circ}$ (ranged, $15^{\circ}-20^{\circ}$ ) and the neck shaft angle was $125^{\circ}$ (ranged, $110^{\circ}-140^{\circ}$ ), indicating normal anatomical relation between the head and neck of hip (Figure 1). Among the patients associated with the femoral head and/or neck defect, 2 patients returned to normal femoral morphology, 17 patients showed malunion of femoral head, and 3 patients with femoral neck stump acted
Table 2 Clinical outcome of 24 patients at 2 years after surgery

\begin{tabular}{ll}
\hline Follow-up study & Patients \\
\hline Duration (years) & 3 (ranged, 2-5) \\
Hip joint reduction & 21 \\
Concentric reduction & 3 \\
Off-concentric reduction & \\
Acetabular index & 22 \\
$15^{\circ}-20^{\circ}$ & 2 \\
$25^{\circ}-30^{\circ}$ & \\
Femoral morphology & 2 \\
Normal & 17 \\
Malunion of femur head & 3 \\
Normal reduction of formal neck and acetabulum & \\
Function of the hip joint & 8 \\
Normal & 13 \\
Mild limitation of flexion and rotation & 3 \\
Fibrous ankylosis
\end{tabular}

as femoral head presented a normal relationship of formal head and acetabula (Figure 2).

The hip stability and weight-bearing were improved significantly. Hip pain was relieved. Twelve patients had residual mild lameness and four had moderate lameness, displaying leg length discrepancy (eight patients) with the average of $1.5 \mathrm{~cm}$ (ranged, 1-2 cm). After 2 years postoperatively, 8 patients had normal function of the hip joint; 13 cases had mild limitation of flexion and rotation, and 3 cases had fibrous ankylosis. The Harris hip score was 83 (ranged, 62-90).

\section{Discussion}

Pathological dislocation of a joint is a complication that directly involves the joint surfaces, which is common in the hip joint. Destructive dislocation occurs commonly in tuberculosis of the hip because of a traveling acetabulum (destruction of the acetabulum) or in septic arthritis of the hip in infancy secondary to the total destruction of femur head. Distensile dislocation occurred because of rapid distension of the joint capsule due to effusion of synovial fluid or pus [7].

Since the pathologically dislocated hips generally did not become normal spontaneously during growth, detection at early stage was necessary and then appropriate remedial measures were performed [8,9]. If the abducted femoral head was hinged on the acetabular margin, which was common in subluxated and dislocated hips, spontaneous progressive cephalocotyloid improvement could not be expected without having a non-hinging hip by any measure. Johari et al. has reported the management of hip dislocations [10] and advocated a surgical 


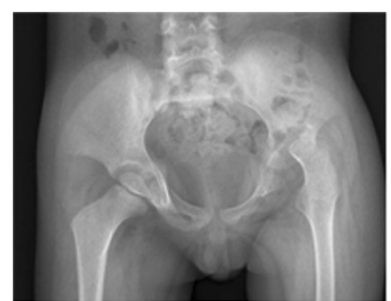

$\mathbf{a}$

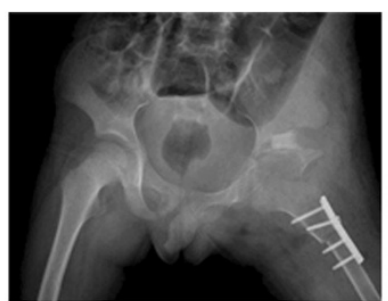

b

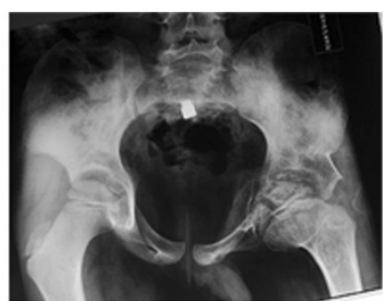

c

Figure 1 Serial radiographs of a 6-year-old boy who presented with tuberculosis of left hip dislocation. He was treated by left open reduction, Pemberton pelvis osteotomy, and corrective osteotomy in the left proximal femur. (a) Pre-operative. (b) Immediate post-operative. (c) 2 years post-operative.

intervention in failed closed reductions and in patients older than 2 years. In this study, we believed that patients even with active tuberculosis also needed aggressive treatment. The operation was performed after antitubercular chemotherapy for 2-4 weeks.

Davidson and Fernandez [11] have reported six cases of the dislocating type out of 31 cases of tuberculous hip (almost 20\% incidence) in children younger than 14 years, and all 6 cases had poor outcomes. However, Campbell and Hoffman [12] have reported 13 cases of dislocating type of 74 hips (about 17\% incidence), and the outcome was excellent/good in $46 \%$ of these cases (6 of 13). A poor outcome was found in those patients who had a narrow joint space after reduction. However, in the present study, all patients achieved satisfied outcomes which might be due to the different surgical procedures we adopted based on the age and the region, range, or extent of pathological dislocation.

Zadeh et al. [13] have reported the management of developmental hip dislocations and advocated that the age at operation might be a significant prognostic factor in the long follow-up, suggesting that $94 \%$ of patients under the age of 2 years achieved an acceptable outcome, while the proportion of patients aged 2-4 years and more than 4 years was $80 \%$ and $71 \%$, respectively. Johari et al. [10] have reported a surgical intervention in failed closed reductions in patients older than 2 years and emphasized the need of supplemental procedures such as Dega osteotomy, Staheli shelf procedure, and femoral varus/derotational osteotomies combined with open reduction. Consistent with prior studies, in this study, patients under the age of 2 years were treated with open reduction associated with external fixation and the outcome was well.

Acetabular reconstruction is essential for patients with severe destruction of acetabulum and femoral head-neck as well as low stability of articular reduction. However, young age, the extent of coverage of the acetabular component by the graft, and secure fixation and mechanical loading of the graft might be impact factors $[14,15]$. Salter osteotomy was only fit for children aged 1-6 years with well-developed acetabular. Meanwhile, femoral head of these patients should be generally congruent with their acetabular roof. Salter osteotomy can provide a limited correction $\left(15^{\circ}-20^{\circ}\right)$ which can be considered as a disadvantage in patients with severe dysplasia $[16,17]$. For children younger than 18 months, Salter osteotomy was contraindicated because satisfactory outcomes could be achieved by other measures [18]. Besides, the patients were usually too small to harvest or fix bone graft. For children older than 6 years, the prerequisites of complete reduction and reasonable congruity usually could not be met, hence it was contraindicated. Pemberton osteotomy was usually performed in patients aged 3-6 years with slightly shallower acetabulum and severe acetabular deficiency and was the most widely known incomplete transiliac osteotomy. Pemberton osteotomy starts approximately

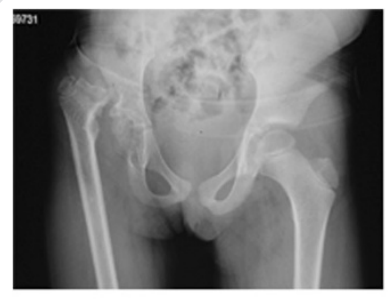

$\mathbf{a}$

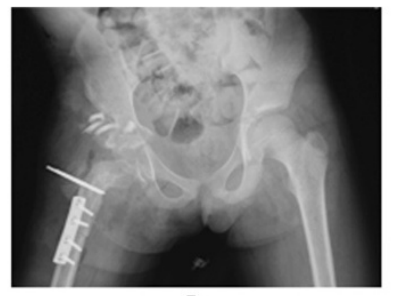

b

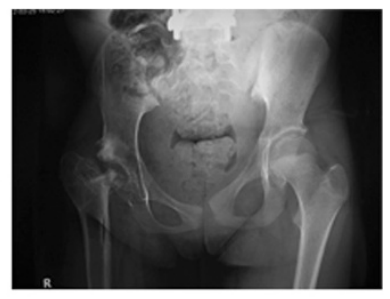

c

Figure 2 Serial radiographs of an 11-year-old girl who presented with tuberculosis of hip dislocation, thoracolumbar kyphosis, and spinal tuberculosis. She was treated by right open reduction, Dega pelvis osteotomy, and subtrochanteric shortening osteotomy in the left proximal femur. (a) Pre-operative. (b) Immediate post-operative. (c) 2 years post-operative. 
10 to $15 \mathrm{~mm}$ above the anterior inferior iliac spine and curves gently posteriorly; the outer and inner tables of the ilium were osteotemized separately and ended at the level of the ilioischial limb of the triradiate cartilage halfway between the sciatic notch and the posterior part of the acetabular rim. Dega osteotomy was also a reshaping, incomplete osteotomy. The theoretical location of the hinge in the Dega osteotomy can involve, to varying degrees, the sciatic notch, the posterior portion of the inner pelvic cortex, the horizontal limb of the triradiate cartilage, and the symphysis pubis [19]. Similar to Pemberton osteotomy, Dega osteotomy also increases acetabular volume.

Subtrochanteric shortening osteotomy is a safe and predictable procedure to restore the anatomic hip center and trochanteric rotation without neurologic injury in high developmental hip dislocation [20]. It allows acetabular exposure, diaphyseal shortening, and facilitates femoral derotation. In this study, although the femoral head was nearly completely absent, the joint function of patients restored to an adequate level by placing stump of femur neck inside the acetabulum. For cases with coxa vara, derotational femoral shortening was performed combined with corrective osteotomy.

Many scholars have always disputed whether internal fixator could be left in the affected hip joint with pathological dislocation. Some researchers believe that using internal fixator will increase numerous of risk such as infection, recrudescence, foreign body reaction, and formation of fistula. We hold that the internal fixator should not be adopted in chronic inflammation infection period. Our clinical data indicated that using the limited internal fixation could greatly enhance the strength of acetabular bone after reconstruction. In addition, prompt removal of the internal fixator had not precluded wound from heal up. In the present study, we did not find any case that had complications caused by internal fixator.

\section{Conclusions}

We have good results for surgical treatments in 29 patients with pathologic dislocation of tuberculous hip. Individualized surgical approach should be taken to treat tuberculosis hip in adolescent patients according to the pathological changes of affected acetabulum. In addition, based on this experience, the use of the early reconstructive surgery of the hip appeared effective even for patients with severely damaged acetabulum.

\section{Competing interests}

The authors declare that they have no competing interests.

\section{Authors' contributions}

$\mathrm{XJ}$ and $\mathrm{YL}$ carried out the studies, participated in the sequence alignment, and drafted the manuscript. LL carried out the immunoassays with XY. MP participated in the sequence alignment. XY participated in the design of the study and performed the statistical analysis. DW conceived of the study, participated in its design and coordination, and helped to draft the manuscript. All authors read and approved the final manuscript.

\section{Acknowledgement}

Applied basic research programs funded projects in Sichuan province (2012JY0086): iatrogenic epiphyseal plate penetrating wound on the developmental effects of children's epiphyseal plate.

Received: 22 October 2013 Accepted: 19 February 2014 Published: 10 March 2014

\section{References}

1. Fabry G: Clinical practice. Eur J Pediatr 2010, 169:143-148.

2. Wang $Y$, Wang J, Xu Z, Li Y, Wang H: Total hip arthroplasty for active tuberculosis of the hip. Int Orthop 2010, 34:1111-1114.

3. Masood S: Diagnosis of tuberculosis of bone and soft tissue by fineneedle aspiration biopsy. Diagn Cytopathol 1992, 8:451-455.

4. Haddad R, Cook SD, Brinker MR: A comparison of three varieties of noncemented porous-coated hip replacement. J Bone Joint Surg Br 1990, 72:2-8.

5. Harris WH: Traumatic arthritis of the hip after dislocation and acetabular fractures: treatment by mold arthroplasty. An end-result study using a new method of result evaluation. J Bone Joint Surg 1969, 51:737-755.

6. Kleinberg S, LIEBERMAN HS: The acetabular index in infants in relation to congenital dislocation of the hip. Arch Surg 1936, 32:1049.

7. Tahasildar N, Sudesh P, Tripathy SK, Shashidhar B: Bilateral pathological dislocation of the hip secondary to tuberculous arthritis following disseminated tuberculosis: a case report and review of the literature. J Pediatr Orthop B 2012, 21:567-573.

8. Moon M-S, Rhee S-K, Lee K-S, Kim S-S: A natural course of a dislocated healed tuberculous hip in a child: a case report. Clin Orthop Relat Res 1984, 190:154-157.

9. Mousa H-L: Evaluation of sinus-track cultures in chronic bone infection. J Bone Joint Surg Br 1997, 79:567-569.

10. Johari AN, Dhawale AA, Johari RA: Management of post septic hip dislocations when the capital femoral epiphysis is present. J Pediatr Orthop B 2011, 20:413-421.

11. Davidson PT, Fernandez E: Bone and joint tuberculosis. Pennsylvania State University College of Medicine, Praeger Publishing: In Monograph in Tuberculosis. Edited by Schlosery D; 1983:119-131.

12. Campbell J, Hoffman E: Tuberculosis of the hip in children. J Bone Joint Surg Br 1995, 77:319-326.

13. Zadeh H, Catterall A, Hashemi-Nejad A, Perry R: Test of stability as an aid to decide the need for osteotomy in association with open reduction in developmental dysplasia of the hip a long-term review. J Bone Joint Surg Br 2000, 82:17-27.

14. Kim M, Kadowaki T: High long-term survival of bulk femoral head autograft for acetabular reconstruction in cementless THA for developmental hip dysplasia. Clin Orthop Relat Res 2010, 468:1611-1620

15. Spangehl MJ, Berry DJ, Trousdale RT, Cabanela ME: Uncemented acetabular components with bulk femoral head autograft for acetabular reconstruction in developmental dysplasia of the hip results at five to twelve years. J Bone Joint Surg Br 2001, 83:1484-1489.

16. Utterback TD, MacEwen GD: Comparison of pelvic osteotomies for the surgical correction of the congenital hip. Clin Orthop Relat Res 1974, 98:104-110.

17. Wong-Chung J, Ryan M, O'Brien TM: Movement of the femoral head after Salter osteotomy for acetabular dysplasia. J Bone Joint Surg Br 1990, 72:563-567.

18. Pekmezci M, Yazici M: Salter osteotomy: an overview. Acta Orthop Traumatol Turc 2004, 41:37-46.

19. Mubarak S, Valencia F, Wenger D: One-stage correction of the spastic dislocated hip. Use of pericapsular acetabuloplasty to improve coverage. J Bone Joint Surg Am 1992, 74:1347-1357.

20. Masonis JL, Patel JV, Miu A, Bourne RB, McCalden R, MacDonald SJ, Rorabeck CH: Subtrochanteric shortening and derotational osteotomy in primary total hip arthroplasty for patients with severe hip dysplasia: 5-year follow-up. J Arthroplasty 2003, 18:68-73.

doi:10.1186/1749-799X-9-16

Cite this article as: Jiang et al:: Pathological dislocation of the hip due to coxotuberculosis in children: a 29-case report. Journal of Orthopaedic Surgery and Research 2014 9:16. 